\title{
Effect of Growth Cycles on the Luminescence Properties of InP/InGaP Quantum Structures Grown Using Migration-Enhanced Epitaxy
}

Received August 27, 2019; revised September 27, 2019; accepted September 30, 2019

II-Wook Cho ${ }^{a}$, Mee-Yi Ryu ${ }^{\mathrm{a}, *}$, and Jin Dong Song ${ }^{\mathrm{b}}$

aDepartment of Physics, Kangwon National University, Chuncheon 24341, Republic of Korea

${ }^{b}$ Center for Opto-Electronic Convergence Systems, Korea Institute of Science and Technology, Seoul 02792, Republic of Korea

*Corresponding author E-mail: myryu@kangwon.ac.kr

\begin{abstract}
The luminescence properties of InP/InGaP quantum structures (QSs) grown using migration-enhanced epitaxy (MEE) with different numbers of repetition of the growth cycles are investigated using temperature-dependent photoluminescence (PL) and time-resolved PL. As the number of MEE repetition cycles increases from 3 to 8, the main PL peak originating from quantum dashes (QDashes) is red-shifted from 1.74 to $1.59 \mathrm{eV}$, owing to the change in the aspect-ratio of InP QDashes, whereas the PL peak energy originating from InP quantum dots and InGaP wetting layer remains nearly constant. The sample grown with six MEE repetition cycles shows the strongest integrated PL intensity with a comparatively narrow linewidth at room temperature. The six-cycled sample demonstrates an increase in PL decay time and radiative lifetime up to the highest temperature $(120 \mathrm{~K})$. These results indicate that the structural and luminescence properties of the InP/InGaP QSs can be controlled by adjusting the number of MEE repetition cycles; the best optical properties are obtained with the six-cycled InP/InGaP QSs sample at a growth temperature of $480^{\circ} \mathrm{C}$.
\end{abstract}

Keywords: Quantum structure, Photoluminescence, Time-resolved photoluminescence, Migration-enhanced epitaxy

\section{Introduction}

Low-dimensional semiconductor quantum structures (QSs) such as quantum dots (QDs) and quantum dashes (QDashes) have attracted considerable research attention as prospective materials for use in light-emitting devices, optical amplifiers, solar cells, and transistors [1-3]. InP/InGaP QSs have been studied for manufacturing high-power light sources with an emission wavelength below $850 \mathrm{~nm}$ [4-8]. A migration-enhanced epitaxy (MEE) method was employed to enhance the uniformity and alignment of QSs [9-11]. The structural and optical properties of InP/InGaP QSs grown using the MEE method were affected by growth temperature, repetition number of growth cycle, interruption time, InGaP spacer thickness, and others [10-14]. In particular, the structural and luminescence properties of InP/InGaP QSs grown with different numbers of MEE repetition cycles were characterized using atomic force microscopy (AFM), scanning electron microscopy (SEM), photoluminescence (PL), and emission wavelength-dependent time-resolved PL (TRPL) [10,12]. However, merely understanding the luminescence properties and carrier dynamics of InP/InGaP quantum structures (QSs; QDs + QDashes) grown with different numbers of MEE repetition cycles is not sufficient.

In this paper, we investigated the luminescence properties and recombination mechanisms of $\operatorname{InP} / \mathrm{InGaP}$ QSs as a function of the number of MEE repetition cycles using temperature-dependent PL and TRPL spectroscopies. As the number of MEE repetition cycles increases from 3 to 8 , the main PL peak originated from QDashes was red-shifted, owing to the increase in the size (width and height) of QDashes. The six-cycled sample exhibited the strongest integrated PL intensities and the increase in the PL decay time up to the highest temperature $(120 \mathrm{~K})$.

\section{Experimental details}

InP/InGaP QS samples were grown on semi-insulating GaAs substrates using a solid source MBE system. Prior to the growth of InP QSs, a $0.1-\mu \mathrm{m}$-thick GaAs buffer layer and a 50 -nm-thick InGaP spacer layer were deposited on the GaAs substrates at temperatures of $580{ }^{\circ} \mathrm{C}$ and $480{ }^{\circ} \mathrm{C}$, respectively. For the growth of InP/InGaP QS samples, the MEE method wherein In and $\mathrm{P}_{2}$ sources are alternately supplied with a growth interruption (GI) time was used. The GI time between depositions was provided to obtain the migration time of sources. One cycle in the MEE growth method consisted of In and $\mathrm{P}_{2}$ sources for a 2 $\mathrm{s}$ supply and a GI for $10 \mathrm{~s}$ after supplying each source. InP QSs used in this study were grown with different numbers of repetition cycles ( 3 to 8 cycles) at a temperature of $480{ }^{\circ} \mathrm{C}$. More information for the growth conditions and MEE method can be found in Ref. 10.

PL measurements were employed using a spectrometer (DM500i, Dongwoo Optron Co., Ltd.), a 532-nm diode laser (MGL-FN-532, CNI Laser) as an excitation source, and a charge coupled device detector 
(ANDOR DV420-BU2). The samples were mounted in a closed-cycle refrigerator (CCS-150, Janis Research Co., Inc.), which recirculate a fixed volume of helium gas to provide cooling to the sample mounting stages, and temperatures ranging from 10 to $300 \mathrm{~K}$ were adjusted by a cryogenic temperature controller (331, Lake Shore Cryotronics, Inc.). In order to verify the recombination dynamics, temperature-dependent TRPL measurement were carried out using a spectrometer (FLS 920, Edinburgh Instruments Ltd.), a picosecond-pulsed diode laser $(\lambda=$ $375 \mathrm{~nm}$, pulse width $=90$ ps, EPL-375, Edinburgh Instruments Ltd.) as an excitation source, and a micro-channel-plate photomultiplier tube detector using a time-correlated single photon counting system. For the temperature-dependent TRPL measurements, the samples were mounted in a closed-cycle cryostat (GB15, Cryomech Inc.) and a temperature controller (ITC503S, Oxford Instruments) were used for monitoring temperatures $(10-300 \mathrm{~K})$.

\section{Results and discussion}

Figure 1(a) displays the room temperature (RT) PL spectra of InP/InGaP QS samples grown with different numbers of repetition of the MEE growth cycle. The PL spectra of all samples include three PL peaks comprising a broad strong peak (P1) and two shoulder peaks that appear below and above P1 (P2 and P3, respectively). Peaks P1 and $\mathrm{P} 2$ are attributed to the emissions of InP QDashes and QDs, respectively, and peak $\mathrm{P} 3$ is ascribed to the emission of the $\mathrm{InGaP}$ wetting layer (WL).

The AFM and SEM images verified the formation of InP QDashes and QDs [10]. The PL peak energies of InP/InGaP QS samples extracted from Gaussian (P2 and P3) or exponentially modified Gaussian (P1) fitting are shown in the inset of Fig. 1(a). With an increase in the number of MEE repetition cycles from 3 to 8 , the peak energy of P1 gradually decreases from 1.74 to $1.59 \mathrm{eV}$ and that of P2 slightly changes between 1.50 and $1.53 \mathrm{eV}$, as seen in the inset of Fig. 1(a). In contrast, the peak energy of $\mathrm{P} 3$ remains unchanged as the number of repetition cycles increases. The redshift of $\mathrm{P} 1$ can be ascribed to the
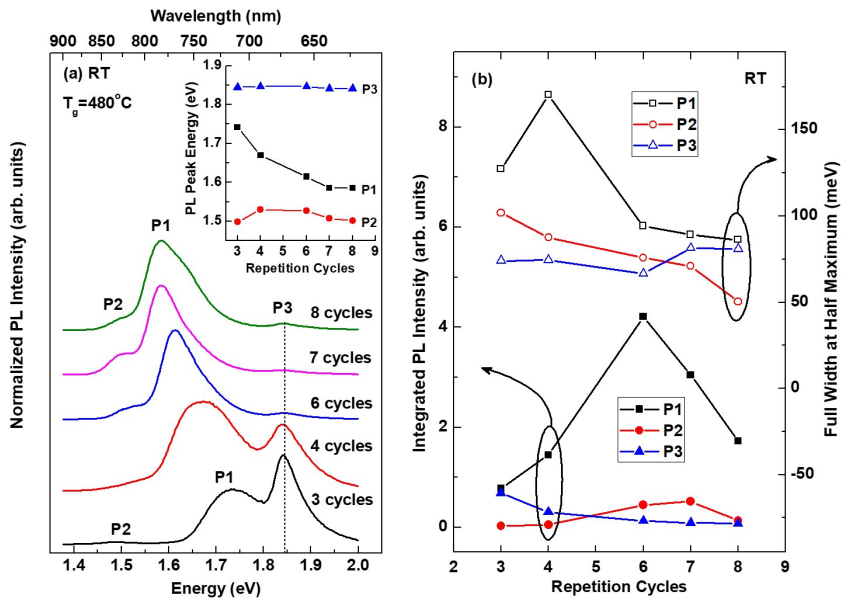

Figure 1. (Color online) (a) PL spectra of $\ln P / \ln G a P$ QS samples grown with different numbers of MEE repetition cycles (3 to 8 cycles) measured at room temperature (RT). The PL emission peak located at intermediate, lower, and higher energies are denoted as P1, P2, and P3, respectively. The inset shows the PL peak energies of $\ln P / I n G a P$ QS samples as a function of the number of MEE repetition cycles. (b) The integrated PL intensities and FWHMs of InP//nGaP QS samples are shown as a function of the number of MEE repetition cycles. change in the aspect-ratio of InP QDashes. In the SEM and AFM images, the height and width of QDashes were seen to be increased because of an increase in the indium amount caused by the increase in the number of repetition cycles while the length of QDashes was shortened [10]. Therefore, an increase in quantum size caused by increasing the number of MEE repetition cycles leads to a decrease in the peak energy of $\mathrm{P} 1$.

Figure 1(b) shows the integrated PL intensity and full width at half maximum (FWHM) of PL peaks (P1, P2, and P3) as a function of the number of MEE repetition cycles. The integrated PL intensity of P3 slowly decreases with increasing the number of repetition cycles. On the other hand, the integrated PL intensities of P1 and P2 exhibit an increase and decrease as the number of MEE repetition cycles increases from 3 to 8 . The increase in the PL intensities can be explained by the increase in the density of QSs; the decrease in the PL intensities during the repetition cycles of 7 and 8 can be attributed to defects or lattice mismatch between the InP and InGaP layers [10]. As the number of MEE repetition cycles increases from 3 to 8 , the increase and decrease in the densities of QDashes and QDs, respectively, were demonstrated by the AFM image [10]. The FWHM of P1 peak is remarkably decreased from 170 to $94 \mathrm{meV}$ as the number of MEE repetition cycles increase from 4 to 6 , and then, it becomes slightly narrow up to $86 \mathrm{meV}$. This FWHM behavior can be explained by the uniform growth of InP/InGaP QSs for MEE repetition cycles from 6 to 8 . The 6-cycled sample exhibits the strongest integrated PL intensity and relatively narrow FWHM.

To investigate the PL decay dynamics of InP/InGaP QS samples grown with various MEE cycles, TRPL measurements were performed as a function of temperature from 10-180 K. Figures 2(a)-2(c) show the temperature-dependent PL decay curves of InP/InGaP QS samples grown with 4,6 , and 8 MEE repetition cycles, respectively. The PL decay curves were plotted at the main PL peak (P1) of each sample. All three samples indicate that the PL decays become slower from 10 to $100-120 \mathrm{~K}$, abruptly shortening as the temperature increases further up to $180 \mathrm{~K}$. The PL decay times of the 4 -cycled $\mathrm{InP} / \mathrm{InGaP}$ QS sample are calculated using the bi-exponential function; $I(t)=A_{1} \exp \left(-t / \tau_{1}\right)+$ $A_{2} \exp \left(-t / \tau_{2}\right)$, where $\tau_{1}$ and $\tau_{2}$ are the fast and slow decay times, respectively, and $A_{1}$ and $A_{2}$ are the pre-exponential constants. The decay times of the 6- and 8-cycled samples are estimated using a single-exponential function. The fast decay time $\tau_{1}$ for the 4 -cycled sample is dominant at low temperatures, and thus, $\tau_{1}$ corresponds to the decay time of the QDashes in the sample. The slow decay time $\tau_{2}$
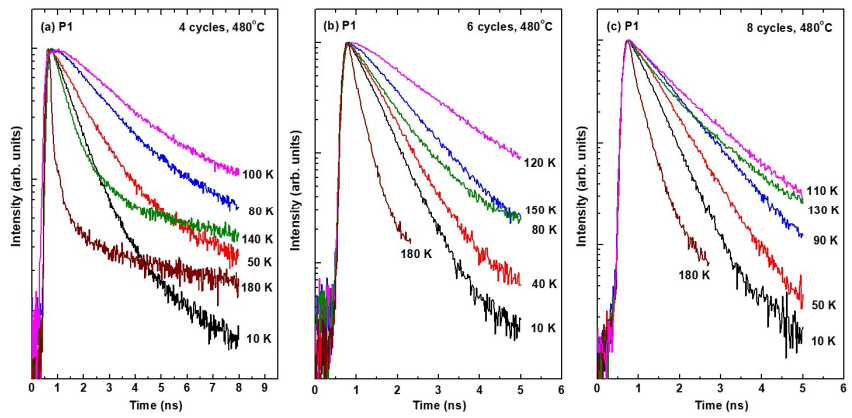

Figure 2. (Color online) Temperature-dependent $P L$ decay curves of $\operatorname{InP} / \mathrm{ln} G a P Q S$ samples grown with (a) 4, (b) 6, and (c) 8 MEE repetition cycles at the P1 peak position for each corresponding temperature. 

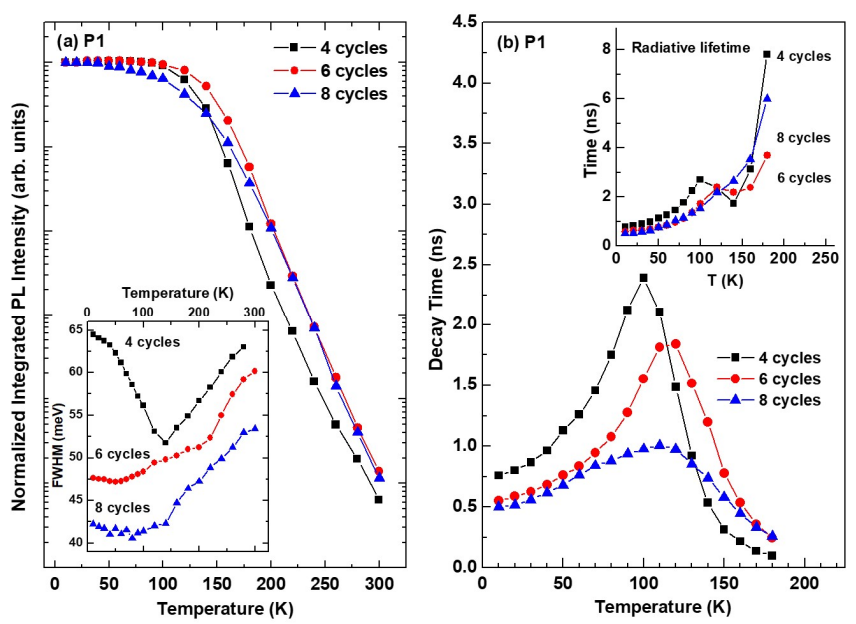

Figure 3. (Color online) (a) Normalized integrated PL intensities and (b) PL decay times of $\mathrm{P} 1$ in $\mathrm{InP} / \mathrm{InGaP} \mathrm{QS}$ samples grown with 4, 6, and 8 MEE repetition cycles as a function of temperature. The temperature-dependent FWHMs and radiative lifetimes of $\mathrm{P} 1$ for $\ln P / \operatorname{lnGaP} Q S$ samples are displayed in the insets of (a) and (b), respectively.

for the 4-cycled sample may be attributed to carrier redistribution from higher energy states (smaller QSs) to lower energy states (larger QSs) via the WL $[12,15,16]$. The decay times $\tau$ of the 6- and 8-cycled samples also correspond with the PL decay times of the QDashes in InP/InGaP QS samples.

The temperature-dependent integrated PL intensities of P1 in InP/InGaP QS samples grown with different numbers of MEE repetition cycles are shown in Fig. 3(a). The integrated PL intensities of all three samples are almost constant up to $\sim 100 \mathrm{~K}$, while decreasing rapidly with an increase in temperature up to $300 \mathrm{~K}$. The PL intensity of the 6-cycled sample is larger than that of the other samples for all temperatures. The temperature-dependent FWHMs of P1 in InP/InGaP QS samples are shown in the inset of Fig. 3(a). As temperature increases from 10 to $140 \mathrm{~K}$, the FWHM of the 4-cycled sample becomes narrower from 64 to $52 \mathrm{meV}$, while beginning to broaden with further increase in temperature up to $300 \mathrm{~K}$. In contrast with the 4-cycled sample, the 6- and 8-cycled samples exhibit a gradual broadening of the FWHM with increasing temperature from 10 to 300 $\mathrm{K}$, indicating the formation of enlarged and/or elongated QDash structures caused by agglomerated QDs with increasing number of MEE repetition cycles.

The FWHM narrowing in the 4-cycled sample is attributed to an increase in the carrier redistributions from smaller QSs to larger QSs with increasing temperature [14]. The decrease in PL intensity and the broadening of FWHM with increasing temperature are attributed to the increase in the non-radiative recombination process and thermal expansion of the carrier distribution function, respectively [17].

Figure 3(b) shows the temperature-dependent PL decay times of InP/InGaP QS samples estimated with the PL decay curves shown in Fig. 2. The PL decay times for all three samples are increased up to 100 $-120 \mathrm{~K}$, and then decreased at higher temperatures. The PL decay time of the 6-cycled sample is increased up to $120 \mathrm{~K}$, which is the highest temperature among samples. The radiative $\left(\tau_{\mathrm{R}}\right)$ and non-radiative $\left(\tau_{\mathrm{NR}}\right)$ lifetimes were calculated with $\eta(T)=\tau_{\mathrm{PL}}(T) / \tau_{\mathrm{R}} \approx I(T) / I_{0}, 1 / \tau_{\mathrm{PL}}=1 / \tau$ ${ }_{\mathrm{R}}+1 / \tau_{\mathrm{NR}}$, where $\eta(T), I(T)$, and $\tau_{\mathrm{PL}}(T)$ are the internal quantum efficiency, integrated PL intensity, and PL decay time, respectively, as a function of temperature. We assumed $\eta(10 \mathrm{~K})=1$ and $I(10 \mathrm{~K})=I_{0}$, and the temperature-dependent integrated PL intensity and PL decay time were used for $I(T)$ and $\tau_{\mathrm{PL}}(T)$, respectively $[18,19]$. In the inset of Fig. 3(b), the estimated radiative lifetimes for all three samples are shown as a function of temperature. As can be seen, the radiative lifetimes for all three samples increase continuously with increasing temperature, which is a typical characteristic of semiconductors. The comparison between the decay time and radiative lifetime indicates that the PL decay times at low temperatures $(\leq 100-120 \mathrm{~K})$ are strongly dependent on radiative recombination. At high temperatures $\left(>100^{-}\right.$ $120 \mathrm{~K}$ ), the decrease in the PL decay time can be attributed to an increase in the non-radiative recombination process. The increase in the decay time at low temperature regions can be explained by several mechanisms, such as thermally activated migration of carriers from $\mathrm{WL}$ and $\mathrm{InGaP}$ barriers, carrier redistribution among the QSs, and reduced wave function overlap caused by carrier expansion.

\section{Conclusions}

The luminescence properties of InP/InGaP QS samples grown with different numbers of repetition of MEE growth cycles were investigated using PL and TRPL spectroscopies as a function of temperature. With an increase in the number of MEE repetition cycles from 3 to 8, the main PL peak originated from QDashes was red-shifted; this was attributed to the increase in the size (width and height) of QDashes. The 6-cycled sample exhibited the strongest integrated PL intensities among the samples at all temperatures ranging from 10 to $300 \mathrm{~K}$. The temperature-dependent PL decay times of InP/InGaP QS samples grown with 4-, 6-, and 8-cycled MEE method showed a typical characteristic of QS semiconductors such, as an increase and decrease in decay time with increasing temperature. The 6-cycled sample demonstrates the strongest PL intensity and the increase in the PL decay time up to the highest temperature (120 K), indicating the optimum number of repetition cycles in the MEE growth at $480{ }^{\circ} \mathrm{C}$ for the formation of InP/InGaP QSs.

\section{Acknowledgements}

This research was supported in part by Basic Science Research Program through the National Research Foundation of Korea (NRF) funded by the Ministry of Education, Science and Technology (NRF2017R1A2B4003744). This study was supported by 2017 Research Grant from Kangwon National University (No. 520170477). Time-resolved photoluminescence measurements were performed at the Central Lab of Kangwon National University.

\section{References}

[1] H. Fariba, W. T. Masselink, and S. H. James, Nanotech. 17, 3703 (2006).

[2] J. P. Reithmaier, A. Somers, S. Deubert, R. Schwertberger, W. Kaiser, A. Forchel, M. Calligaro, P. Resneau, O. Parillaud, S. Bansropun, M. Krakowski, R. Alizon, D. Hadass, A. Bilenca, H. Dery, V. Mikhelashvili, G. Eisenstein, M. Gioannini, I. Montrosset, T. W. Berg, M. v. d. Poel, J. Mørk, and B. Tromborg, J. Phys. D: Appl. Phys. 38, 2088 (2005).

[3] D. Zhou, P. E. Vullum, G. Sharma, S. F. Thomassen, R. Holmestad, T. W. Reenaas, and B. O. Fimland, Appl. Phys. Lett. 
96, 083108 (2010).

[4] G. Walter, N. H. Jr., R. D. Heller, and R. D. Dupuis, Appl. Phys. Lett. 81, 4604 (2002).

[5] A. Ugur, S. Kremling, F. Hatami, S. Höfling, L. Worschech, A. Forchel, and W. T. Masselink, Appl. Phys. Lett. 100, 023116 (2012).

[6] P. M. Smowton, J. Lutti, G. M. Lewis, A. B. Krysa, J. S. Roberts, and P. A. Houston, IEEE J. Sel. Top. Quantum Electron. 11, 10351040 (2005).

[7] J. Lutti, P. M. Smowton, G. M. Lewis, A. B. Krysa, J. S. Roberts, P. A. Houston, Y. C. Xin, Y. Li, and L. F. Lester, Electron. Lett 41, 247 (2005).

[8] S. K. Ha, J. D. Song, I. K. Han, D. Y. Ko, S. Y. Kim, and E. H. Lee, J. Korean Phys. Soc. 59, 3089 (2011)

[9] R. Rödel, A. Bauer, S. Kremling, S. Reitzenstein, S. Höfling, M. Kamp, L. Worschech, and A. Forchel, Nanotech. 23, 015605 (2012).

[10] S. Y. Kim, J. D. Song, I. K. Han, and T. W. Kim, J. Nanosci. Nanotech. 12, 5519 (2012).

[11] H. R. Byun, M. Y. Ryu, J. D. Song, and C. L. Lee, J. Korean Phys.
Soc. 66, 811 (2015).

[12] J. W. Oh, I. W. Cho, M. Y. Ryu, and J. D. Song, Appl. Sci. Converg. Tech. 24, 67 (2015).

[13] I. W. Cho, M. Y. Ryu, and J. D. Song, Appl. Sci. Converg. Tech. 25, 81 (2016).

[14] I. W. Cho, M. Y. Ryu, and J. D. Song, J. Korean Phys. Soc. 70, 785 (2017).

[15] Y. C. Zhang, C. J. Huang, F. Q. Liu, B. Xu, J. Wu, Y. H. Chen, D. Ding, W. H. Jiang, X. L. Ye, and Z. G. Wang, J. Appl. Phys. 90, 1973 (2001).

[16] Y. F. Wu, J. C. Lee, T. E. Nee, and J. C. Wang, J. Lumin. 131, 1267 (2011).

[17] S. Sanguinetti, M. Henini, M. Grassi Alessi, M. Capizzi, P. Frigeri, and S. Franchi, Phys. Rev. B 60, 8276 (1999).

[18] L. M. Kong, J. F. Cai, Z. Y. Wu, Z. Gong, Z. C. Niu, and Z. C. Feng, Thin Solid Films 498, 188 (2006).

[19] J. H. Song, J. W. Lee, P. W. Yu, M. Y. Ryu, J. Zhang, E. Kuokstis, J. W. Yang, and M. Asif Khan, Solid State Commun. 127, 661 (2003). 